\title{
Registration-Based Segmentation Using the Information Bottleneck Method
}

\author{
Anton Bardera, Miquel Feixas, Imma Boada, Jaume Rigau, and Mateu Sbert \\ Institut d'Informàtica i Aplicacions, Universitat de Girona \\ abardera@ima.udg.edu
}

\begin{abstract}
We present two new clustering algorithms for medical image segmentation based on the multimodal image registration and the information bottleneck method. In these algorithms, the histogram bins of two registered multimodal 3D-images are clustered by minimizing the loss of mutual information between them. Thus, the clustering of histogram bins is driven by the preservation of the shared information between the images, extracting from each image the structures that are more relevant to the other one. In the first algorithm, we segment only one image at a time, while in the second both images are simultaneously segmented. Experiments show the good behavior of the presented algorithms, especially the simultaneous clustering.
\end{abstract}

\section{Introduction}

Medical image segmentation plays a crucial role in clinical practice, mainly for diagnosis and disease treatment. It consists in subdividing an image into its constituent parts, a significant step towards image understanding [1. Registration is also a fundamental task in a medical scenario since it allows to combine different image models in a single one in order to enhance data interpretation. In [2] the influence of intensity clustering on mutual information based image registration is studied. On the contrary, the main purpose of this paper is analyze how the segmentation process can benefit from image registration. With this aim, we introduce two clustering algorithms for image segmentation based on the registration of the images to be segmented. These algorithms apply the information bottleneck method 314, which compresses a variable $X$ with minimal loss of mutual information with respect to another variable $Y$.

Given two registered 3D-images, our algorithms work by merging neighbor histogram bins driven by the minimization of the loss of mutual information between the two images. The first algorithm segments just one image at a time, while the second segments both simultaneously. These algorithms provide us with a completely automatic global segmentation method. The intuition behind them is to segment an image $A$ by extracting the structures that are most relevant for another image $B$, i.e., the segmentation of $A$ attempts to preserve the maximum dependence with $B$. Thus, image $B$ controls the segmentation of $A$ and viceversa. Our techniques have been tested on several MR-CT datasets, which have been previously registered using the normalized mutual information [5]. The obtained

J. Martí et al. (Eds.): IbPRIA 2007, Part II, LNCS 4478, pp. 130-137 2007.

(C) Springer-Verlag Berlin Heidelberg 2007 
results show the good behaviour of both segmentation algorithms, especially the simultaneous clustering. This approach can be considered as a first step towards multimodal image visualization.

This paper is organized as follows. In Section 2, some information-theoretic definitions and the information bottleneck method are presented. In Section 3, two new registration-based segmentation algorithms are introduced. In Section 4, experimental results show the suitability of the presented algorithms. Finally, our conclusions are given in Section 5 .

\section{Information Theoretic Tools}

We review some basic concepts of information theory [6], the information bottleneck method [34], and the application of mutual information to image registration [78].

Entropy. The Shannon entropy $H(X)$ of a discrete random variable $X$ with values in the set $\mathcal{X}=\left\{x_{1}, x_{2}, \ldots, x_{n}\right\}$ is defined as $H(X)=-\sum_{i=1}^{n} p_{i} \log p_{i}$, where $n=|\mathcal{X}|, p_{i}=\operatorname{Pr}\left[X=x_{i}\right]$. The logarithms are taken in base 2 and therefore entropy is expressed in bits.

Mutual information. Given two discrete random variables, $X$ and $Y$, with values in $\mathcal{X}=\left\{x_{1}, \ldots, x_{n}\right\}$ and $\mathcal{Y}=\left\{y_{1}, \ldots, y_{m}\right\}$, respectively, the mutual information (MI) between $X$ and $Y$ is defined as

$$
I(X, Y)=\sum_{i=1}^{n} \sum_{j=1}^{m} p_{i j} \log \frac{p_{i j}}{p_{i} q_{j}}
$$

where $n=|\mathcal{X}|, m=|\mathcal{Y}|, p_{i}=\operatorname{Pr}\left[X=x_{i}\right]$ and $q_{j}=\operatorname{Pr}\left[Y=y_{j}\right]$ are the marginal probabilities, and $p_{i j}=\operatorname{Pr}\left[X=x_{i}, Y=y_{j}\right]$ is the joint probability. MI is a measure of the shared information between $X$ and $Y$ [6]. A fundamental property of $\mathrm{MI}$ is the data processing inequality which can be expressed in the following way: if $X \rightarrow Y \rightarrow Z$ is a Markov chain, i.e., $p(x, y, z)=p(x) p(y \mid x) p(z \mid y)$, then

$$
I(X, Y) \geq I(X, Z) \text {. }
$$

This result demonstrates that no processing of $Y$ can increase the information that $Y$ contains about $X[6]$.

Jensen-Shannon divergence. A convex function on the interval $[a, b]$, fulfils that $\sum_{i=1}^{n} \lambda_{i} f\left(x_{i}\right)-f\left(\sum_{i=1}^{n} \lambda_{i} x_{i}\right) \geq 0$, where $0 \leq \lambda \leq 1, \sum_{i=1}^{n} \lambda_{i}=1$, and $x_{i} \in[a, b]$. For a concave function, the inequality is reversed. If $f$ is substituted by the Shannon entropy, which is a concave function, we obtain the Jensen-Shannon inequality [9]:

$$
J S\left(\Pi_{1}, \ldots, \Pi_{n}\right)=H\left(\sum_{i=1}^{n} \pi_{i} \Pi_{i}\right)-\sum_{i=1}^{n} \pi_{i} H\left(\Pi_{i}\right) \geq 0,
$$

where $J S\left(\Pi_{1}, \ldots, \Pi_{n}\right)$ is the Jensen-Shanon divergence of probability distributions $\Pi_{1}, \Pi_{2}, \ldots, \Pi_{n}$ with prior probabilities or weights $\pi_{1}, \pi_{2}, \ldots, \pi_{n}$, fulfill$\operatorname{ing} \sum_{i=1}^{n} \pi_{i}=1$. The Jensen-Shannon divergence is identical to $I(X, Y)$ when 
$\left\{\pi_{i}\right\}$ is the marginal probability distribution $\left\{p_{i}\right\}$ of $X$ and $\left\{\Pi_{i}\right\}$ are the rows $\{p(Y \mid i)\}$ of the conditional probability matrix of the information channel, i.e., $p(Y \mid i)=\left\{p_{1 \mid i}, p_{2 \mid i}, \ldots, p_{m \mid i}\right\}$.

Information bottleneck method. The objective of the information bottleneck method, introduced by Tishby et al. [3, is to extract a compact representation of the variable $X$, denoted by $\widehat{X}$, with minimal loss of MI with respect to another variable $Y$, i.e., $\widehat{X}$ preserves as much information as possible about the relevant variable $Y$. Soft [3] and hard [4 partitions of $X$ can be adopted. In the first case, every cluster $x \in X$ can be assigned to every cluster $\widehat{x} \in \widehat{X}$ with some conditional probability $p(\widehat{x} \mid x)$ (soft clustering). In the second case, every cluster $x \in X$ is assigned to only one cluster $\widehat{x} \in \widehat{X}$ (hard clustering). Our approach is based on this case, also called agglomerative information bottleneck method 4 .

MI-based image registration. Successful image registration methods are based on the maximization of mutual information between two images [78]. The registration of two images can be represented by an information channel $X \rightarrow Y$, where the random variables $X$ and $Y$ represent the images. Their marginal probability distributions, $\left\{p_{i}\right\}$ and $\left\{q_{j}\right\}$, and the joint probability distribution, $\left\{p_{i j}\right\}$, are obtained by simple normalization of the marginal and joint intensity histograms of the overlapping areas of both images [7]. The registration method based on the maximization of MI 78$]$ is based on the conjecture that the correct registration corresponds to the maximum MI between the overlapping areas of the two images. Later, Studholme et al. [5] proposed a normalization of mutual information defined by

$$
N M I(X, Y)=\frac{I(X, Y)}{H(X, Y)},
$$

where $H(X, Y)$ is the joint entropy. NMI is more robust than MI, due to its greater independence of the overlap area.

\section{Clustering Algorithms}

In this section, two clustering algorithms based on the registration of images A and B are introduced. First, we present a greedy hierarchical clustering algorithm [4 that clusters the histogram bins of image $A$ by preserving the maximum MI between A and B. Second, we present a similar algorithm which simultaneously clusters the two images.

\subsection{One-Sided Clustering Algorithm}

In a preprocessing step, images $\mathrm{A}$ and $\mathrm{B}$ are registered, establishing a discrete information channel $X \rightarrow Y$, where $X$ and $Y$ denote, respectively, the histograms of $A$ and B. From the data processing inequality (2), we know that any clustering over $X$ (for instance, merging neighbour histogram bins $x_{i}$ and $x_{i+1}$ ), denoted by $\widehat{X}$, will reduce $I(X, Y)$. Thus, $I(\widehat{X}, Y) \leq I(X, Y)$. 
At the initial stage of our algorithm, only one intensity value is assigned to each cluster $\widehat{x}$ (or bin) of $X$. Then, the algorithm proceeds greedily by merging two neighbour clusters so that the loss of MI be minimum. This procedure merges the two clusters which are more similar from the perspective of B. Note the constraint that only neighbour bins or clusters can be merged. The cardinality $|\widehat{X}|$ of $\widehat{X}$ goes from $|X|$ to 1 .

The efficiency of this algorithm can be greatly improved if the reduction of MI due to the merging of clusters $\widehat{x}_{i}$ and $\widehat{x}_{i+1}$ [4] is computed by

$$
\delta I_{\widehat{X}}=\left(p\left(\widehat{x}_{i}\right)+p\left(\widehat{x}_{i+1}\right)\right) J S\left(p\left(Y \mid \widehat{x}_{i}\right), p\left(Y \mid \widehat{x}_{i+1}\right)\right),
$$

where $J S\left(p\left(Y \mid \widehat{x}_{i}\right), p\left(Y \mid \widehat{x}_{i+1}\right)\right)$ is the Jensen-Shannon divergence (3) and $p\left(Y \mid \widehat{x}_{i}\right)$ denotes the row $i$ of the conditional probability matrix of the information channel. The evaluation of $\delta I_{\widehat{X}}$ for each pair of clusters is done in $O(|Y|)$ operations and, at each iteration of the algorithm, it is only necessary to compute the $\delta I_{\widehat{X}}$ of the new cluster with its two corresponding neighbors [4]. All the other precomputed $\delta I_{\widehat{X}}$ remain unchanged.

Similarly to [10], clustering can be stopped using several criteria: a fixed number of clusters, a given ratio $M I R=I(\widehat{X}, Y) / I(X, Y)$ or a variation $\delta I_{\widehat{X}}$ greater than a given $\epsilon$. The MIR ratio can be considered as a quality measure of the clustering. In the next section, we analyze the behavior of the normalized mutual information, $N M I=I(\widehat{X}, Y) / H(\widehat{X}, Y)$, which provides us with an efficiency coefficient [11] of the segmentation process, and $-\delta I_{\widehat{X}} / I(X, Y)$, which indicates the relative loss of information of a given clustering [3].

\subsection{Co-clustering Algorithm}

It is of interest now to consider a simultaneous clustering of images A and B. Unlike the algorithm presented by Dhillon 12 for word-document clustering, which alternatively clusters the variables $\widehat{X}$ and $\widehat{Y}$, our algorithm (see Fig. 1) chooses at each step the best merging of one of the two images, i.e., the one that entails a minimum reduction of MI. The similarity between the two images is being symmetrically exploited. Thus, each clustering step benefits from the progressive simplification of the images. One of the main advantages of this algorithm is the great reduction of sparseness and noise of the joint probability matrix. As we will see in the next section, the simultaneous merging over the images $\mathrm{A}$ and $\mathrm{B}$ obtain better results than with the one-sided algorithm.

From the data processing inequality (2) $I(\widehat{X}, \widehat{Y})$ is a decreasing function with respect to the reduction of the total number of clusters $|\widehat{X}|+|\widehat{Y}|$. Thus, $I(\widehat{X}, \widehat{Y}) \leq I(X, Y)$. Like the one-sided algorithm, the stopping criterion can be given by a predefined number of bins, a given ratio $M I R=I(\widehat{X}, \widehat{Y}) / I(X, Y)$ or a variation $\delta I_{\widehat{X}}$ (or $\delta I_{\widehat{Y}}$ ) greater than a given $\epsilon$. Similarly to the above onesided algorithm, the reduction of MI can be computed from the Jensen-Shannon divergence (5). But in the co-clustering algorithm, for each clustering of $\widehat{X}$ (or $\widehat{Y})$, it is necessary to recompute all the $\delta I_{\widehat{Y}}\left(\right.$ or $\delta I_{\widehat{X}}$ ). Figure 1 shows the coclustering algorithm where the stopping criterion is given by the total number of clusters. 


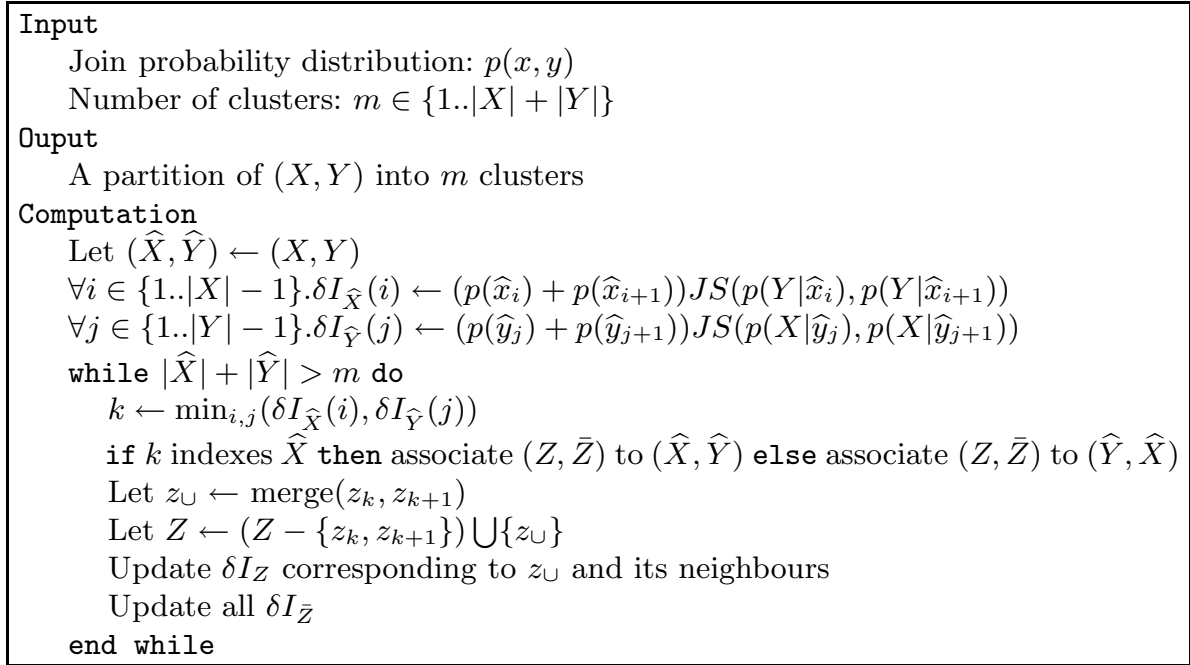

Fig. 1. Co-clustering algorithm

\section{Results and Discussion}

To evaluate the performance of the proposed algorithms, the results of two different patients from the Vanderbilt database are shown. Both datasets are composed of MR and CT image modalities. The resolution of the MR and CT is $256 \times 256 \times 26$ and $512 \times 512 \times 28$, respectively. For each patient, MR and CT images have been registered using the $N M I$ measure [5].

In Fig. 2, we show the results obtained with the one-sided and co-clustering algorithms applied on the CT (Fig. 2( $(i i . a)$ ) and MR (Fig. 2( iii.a)) original images of the first dataset. Columns $(b-d)$ show the segmented images with 2,4 , and 6 clusters, respectively. The results obtained with the one-sided algorithm applied on the CT and MR images are shown in Fig. 2 $(i . b-d)$ and Fig. 2( $(i v . b-d)$, respectively. The results obtained with the co-clustering algorithm are shown for the CT image in Fig. 2( $(i i . b-d)$ and for the MR in Fig. 2( $(i i i . b-d)$.

If we compare the original unsegmented images with the resulting segmented images, the following is observed. First, we can see that the best results are obtained with the co-clustering algorithm (Fig. $2(i i-i i i . b-d)$ ). There is clear evidence that hidden structures of the image are more precisely recovered. Compare, for instance, the images for an equal number of clusters of Fig. 2(i.c) and Fig. 2( ii.c). This better behaviour can be explained because in the co-clustering case we make use of all bidirectional information obtained with the progressive simplification of both images. Second, for both algorithms, results appear much better segmenting the CT images than the MR ones. This is due to the fact that the segmentation of the CT images benefits a lot from the precise information contained in the MR histogram. 


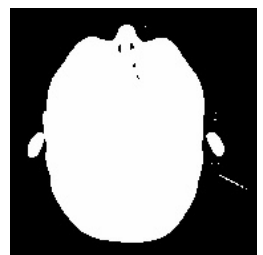

(i.b)

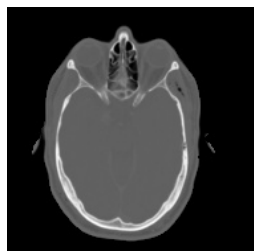

(ii.a)

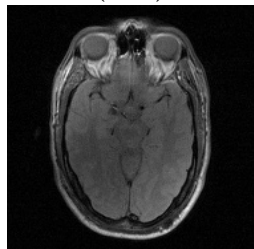

(iii.a)

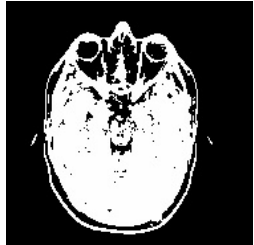

(ii.b)

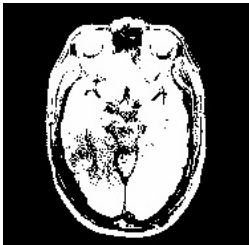

(iii.b)

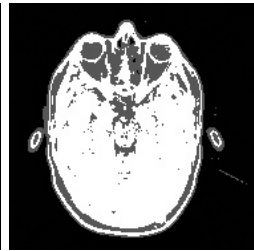

(i.c)

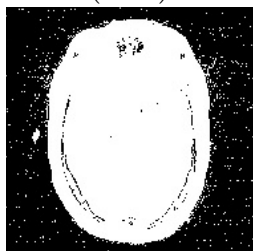

$(i v . b)$

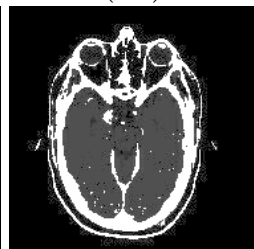

(ii.c)

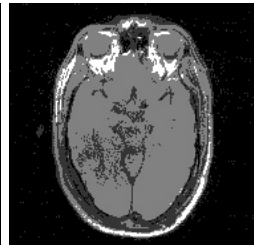

(iii.c)

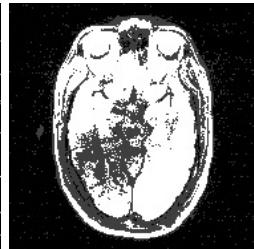

(iv.c)

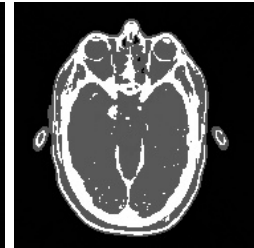

(i.d)

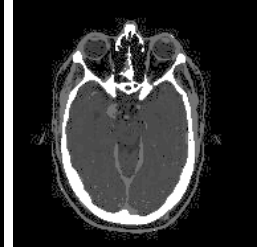

(ii.d)

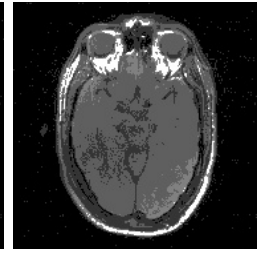

(iii.d)

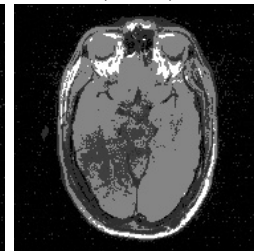

(iv.d)

Fig. 2. (a) Original dataset images. ( $b, c, d)$ Images segmented using 2,4 , and 6 bins, respectively. $(i, i v)$ Images obtained with the one-sided algorithm. (ii, iii) Images obtained with the co-clustering algorithm.

Results of the application of the co-clustering algorithm on the second dataset are illustrated in Fig. 3. MR and CT images, corresponding to two different slices, are shown with 3,4 and 5 clusters. Observe the quality of the resulting images, where anatomical structures are progressively segmented. For instance, in the MR case with 5 clusters, we observe the correct separation of gray matter, white matter, cerebro spinal fluid, skin and background.

Fig. $4(a)$ and Fig. $4(c)$, corresponding to the dataset of Fig. 2. plot the MIR vs the number of clusters for the one-sided and co-clustering algorithms, respectively. We can clearly observe the high quality of the resulting images with a low number of clusters. If the number of clusters decreases below a critical value, MI falls dramatically. On the contrary, to the left of this critical value, MI does 

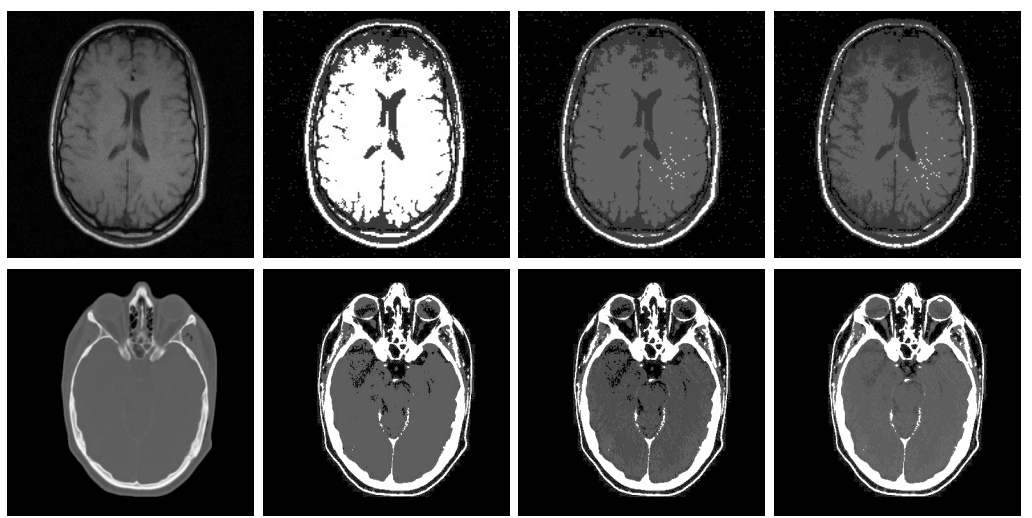

Fig. 3. MR (first row) and CT (second row) original images and their segmentations using the co-clustering algorithm with 3,4 and 5 bins, respectively

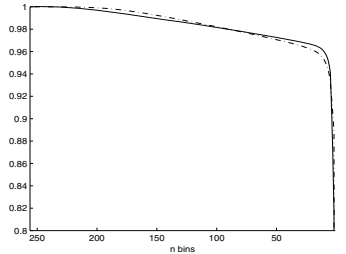

(a)

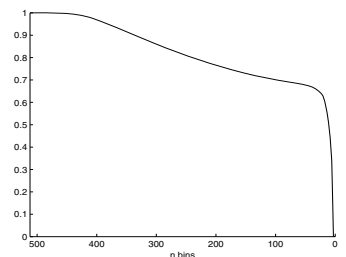

$(c)$

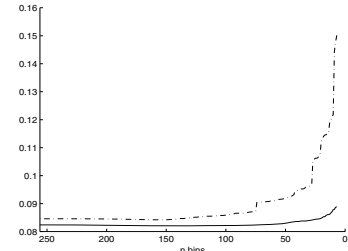

(b)

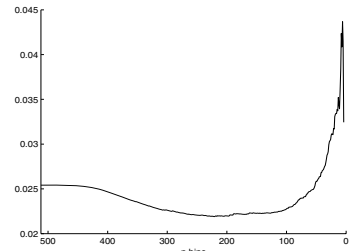

$(d)$

Fig. 4. (a) $M I R$ and (b) $N M I$ vs the number of clusters obtained with the one-sided algorithm applied on CT (solid line) and MR (dashed line) images of Fig. 2 (c) MIR and $(d) N M I$ vs the number of clusters obtained with the co-clustering algorithm.

not increase significantly with the number of clusters. This critical point can be detected by the stopping criterion given by the variation of MI (see Sec. 3).

On Fig. $4(b)$ and Fig. $4(d)$, the efficiency coefficient $N M I$ against the number of clusters for the one-sided and co-clustering algorithms is plotted, respectively. Notice that the efficiency is maximum when the number of bins is low. Comparing both plots, we can see that, while the one-sided algorithm always increases monotonically, in the co-clustering there are fluctuations. This is due to the different decreasing rate of MI and joint-entropy for the co-clustering algorithm. 


\section{Conclusion}

In this paper, we have presented a new image segmentation approach based on the registration of the images and the information bottleneck method. Two algorithms have been presented and analyzed. The first one is a one-sided algorithm which clusters the neighbor bins of only one image based on the minimization of the loss of mutual information between the two images. The second one is a co-clustering algorithm which chooses at each step the best clustering of one of the two images by minimizing the loss of mutual information. Experiments have shown the good behaviour of the presented algorithms. However, it has been shown that the co-clustering algorithm performs better than the one-sided one. In our future work, we will develop a multimodal data visualization framework based on the proposed algorithms.

Acknowledgments. This project has been funded in part with grant numbers TIN2004-08065-C02-02, TIN2004-07451-C03-01 and 2001-SGR-00296.

\section{References}

1. Gonzalez, R.C., Woods, R.E.: Digital Image Processing. Prentice Hall, Upper Saddle River (NJ), USA (2002)

2. Knops, Z., Maintz, J., Viergever, M., Pluim, J.: Normalized mutual information based registration using $\mathrm{k}$-means clustering and shading correction. Medical image analysis 10(3), 432-439 (2006)

3. Tishby, N., Pereira, F.C., Bialek, W.: The information bottleneck method. In: Proceedings of the 37th Annual Allerton Conference on Communication, Control and Computing, pp. 368-377 (1999)

4. Slonim, N., Tishby, N.: Agglomerative information bottleneck. In: Proceedings of NIPS-12 (Neural Information Processing Systems), pp. 617-623. MIT Press, Cambridge, MA (2000)

5. Studholme, C.: Measures of 3D Medical Image Alignment. PhD thesis, University of London, London, UK (1997)

6. Cover, T.M., Thomas, J.A.: Elements of Information Theory. Wiley Series in Telecommunications (1991)

7. Maes, F., Collignon, A., Vandermeulen, D., Marchal, G., Suetens, P.: Multimodality image registration by maximization of mutual information. IEEE Trans. on Medical Imaging 16(2), 187-198 (1997)

8. Viola, P.A.: Alignment by Maximization of Mutual Information. PhD thesis, Massachusetts Institute of Technology, Massachusetts (MA), USA (1995)

9. Burbea, J., Rao, C.: On the convexity of some divergence measures based on entropy functions. IEEE Trans. on Information Theory 28(3), 489-495 (1982)

10. Rigau, J., Feixas, M., Sbert, M., Bardera, A., Boada, I.: Medical image segmentation based on mutual information maximization. In: Barillot, C., Haynor, D.R., Hellier, P. (eds.) MICCAI 2004. LNCS, vol. 3216, Springer, Heidelberg (2004)

11. Butz, T., Cuisenaire, O., Thiran, J.P.: Multi-modal medical image registration: from information theory to optimization objective. In: Proceeding of 14th International Conference on Digital Signal Processing (DSP'02) (2002)

12. Dhillon, I.S., Mallela, S., Modha, D.S.: Information-theoretic co-clustering. In: Proceedings of the 9th ACM SIGKDD 2003, pp. 89-98. ACM Press, NY, USA (2003) 